\title{
Automated Sea Ice Classification Using Spaceborne Polarimetric SAR Data
}

\author{
B. Scheuchl ${ }^{1}$, R. Caves ${ }^{2}$, I. Cumming ${ }^{1}$ and G. Staples ${ }^{3}$ \\ ${ }^{1}$ Dept. of Electrical and Computer Engineering, University of British Columbia, 2356 Main Mall, Vancouver, B.C., Canada \\ V6T 1Z4 E-mail: bernds@ece.ubc.ca, ianc@ece.ubc.ca \\ ${ }^{2}$ MacDonald Dettwiler and Associates, 13800 Commerce Parkway, Richmond, B.C., Canada V6V 2J3 \\ E-mail: rcaves@mda.ca \\ ${ }^{3}$ Radarsat International, 13800 Commerce Parkway, Richmond, B.C., Canada V6V 2J3 \\ E-mail: gstaples@rsi.ca
}

\begin{abstract}
This paper discusses the capability of spaceborne polarimetric C-band SAR data for sea ice detection and classification. Unsupervised classification using polarimetric decomposition and the complex Wishart classifier was performed on SIR-C data acquired off the coast of Newfoundland in April 1994. The algorithm is used for sea ice applications for the first time, and appears promising.

In addition to polarimetric classification, three of the measured features were found to have ice edge detection capability: HV-intensity, HH/VV-ratio and anisotropy. These features show a clear separation between sea ice and open water and simple thresholds can be applied.
\end{abstract}

\section{INTRODUCTION}

Sea ice is a complex composite of a variety of ice types that show strong seasonal variation, and affects ocean traffic routes and offshore operations. Long term changes in the extent of the ice coverage are an indicator of climate change. Accurate and operational monitoring of sea ice is hence important for a better understanding of our planet as well as to improve and secure shipping routes in higher latitudes.

Current spaceborne Synthetic Aperture Radar (SAR) sensors provide operational sea ice monitoring. However, they only provide single polarization data [1]. Dual and fully polarimetric data will become available with the advent of ENVISAT and RADARSAT-2. In this paper, we investigate the improved capability of fully polarimetric data with emphasis on unsupervised classification.

\section{DESCRIPTION OF THE TeST Site}

Our test site is an area in the Labrador Sea off the coast of Newfoundland. Fully polarimetric C-band radar data were acquired on April 18, 1994 during a SIR-C mission. Incidence angles range from $26^{\circ}$ to $31^{\circ}$. Figure 1a shows a false color composite using $\mathrm{HH}, \mathrm{VV}$ and $\mathrm{HV}$ channel intensities. Land, sea ice and open water are present in the scene. The landmass in the upper right of the scene is part of the Gros Morne National Park.

Air temperature records for Rocky Harbour indicate an average temperature above zero degrees for approximately

This work was performed under contract to the Canadian Space Agency (No. 9F028-0-4902/06) as part of the Earth Observation Applications

Development Program. four days with the minimum temperature above zero degrees for 24 hours prior to data acquisition. While a photograph taken from the Shuttle on April 13 shows the ocean fully covered by sea ice, high temperatures and wind caused significantly different conditions for April 18. Surface melt and water saturation of snow can be expected, which in combination with steep incidence angles, makes the SAR scene difficult for sea ice detection and classification.

First year ice (FYI) in various stages of decline can be observed in the scene. A well-defined large floe in the top left of the image shows the strongest return with bright linear structures indicating the presence of ridges or deformed first year ice. The sea ice in the lower half of the image is more chaotic and appears to be severely affected by surface melt and/or saturated wet snow cover resulting in a lower backscatter return. Open water shows strong variation in backscatter strength due to different levels of wind induced surface roughness. Smooth water shows the weakest signal.

The RGB image reveals the high information content of fully polarimetric data. Although it is visually possible to separate sea ice from open water and even distinguish
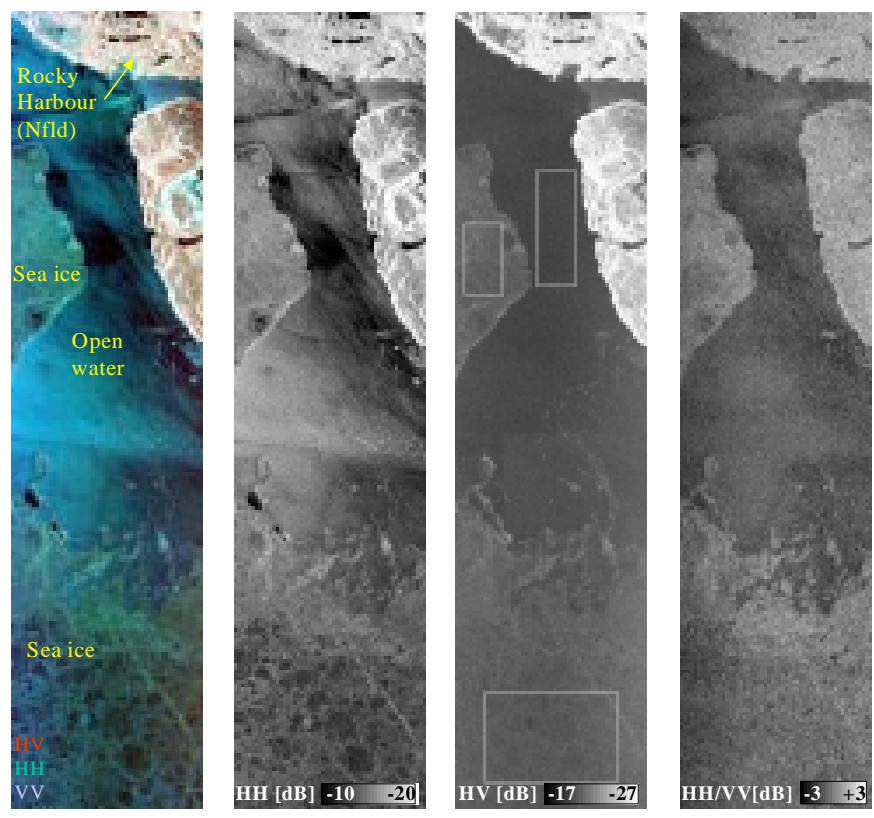

Fig. 1: a) RGB image using the channel intensities, b) $\mathrm{HH}$ image c) HV image with test areas for threshold estimation d) HH/VV ratio image. 
between different ice types, the strong variation in backscatter for sea ice and open water makes classification a challenging task. To reduce the effect of speckle noise the data was multilooked so that one pixel covers an area of about $100 \mathrm{~m}$ x $100 \mathrm{~m}$ (64 looks).

\section{SAR POLARIMETRY}

\section{A. Fully polarimetric data}

By comparing parts (a), (b) and (c) of Figure 1, we can see that polarimetric measurements provide a more complete inference of natural surface parameters than is possible with a single-channel radar system and the potential for ice type differentiation is shown in [2] The scattering matrix is the basic single-look representation of the polarimetric data, whereas the $3 \times 3$ covariance and coherency matrices allow multi-looking of the data on a matrix level [3]. With the scattered wave being a superposition of waves scattered within a resolution element, several different scattering mechanisms can potentially contribute to the reflected signal of each pixel. Target decomposition allows a better physical interpretation of the target with respect to scattering mechanisms [4].

One possible decomposition approach is to perform an eigenvalue decomposition of the coherency matrix. The eigenvectors can be interpreted as scattering mechanisms and the corresponding eigenvalues as their relative strength. Using eigenvectors and eigenvalues, the target can be described using three physically-based rotation-invariant parameters [4]. The entropy, $\mathrm{H}$, is defined by the eigenvalues and describes the randomness of the scattering. $\mathrm{H}=1$ indicates a completely random mixture of scattering mechanisms with equal probability (a completely depolarizing target). $\quad \mathrm{H}=0$ indicates a single scattering mechanism, i.e. isotropic scatterer.
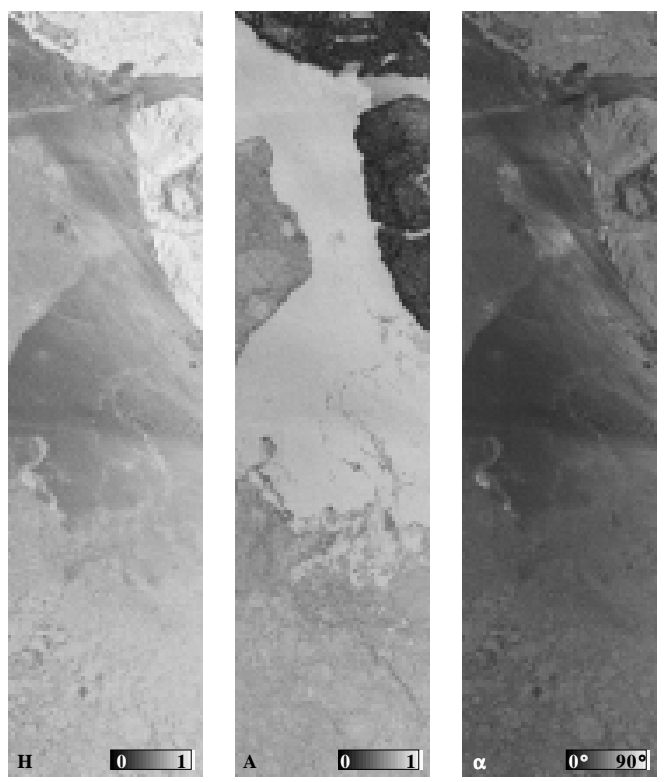

Fig. 2: Entropy H, Anisotropy A and $\alpha$-angle for the scene.
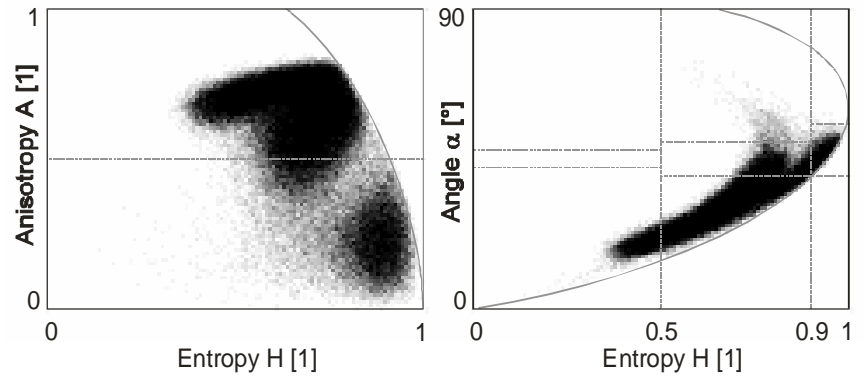

Fig. 3: Entropy / Anisotropy / $\alpha$-angle classification space with partitions and bounds.

The anisotropy represents the relative powers of the second and third eigenvalues. The $\alpha$-angle represents an average dominant scattering mechanism. It ranges from zero (surface scattering) to 90 degrees (dihedral scattering) and is derived using eigenvectors and eigenvalues. The three parameters for the test scene are shown in Figure 2.

The distribution of the data in the H/A and $\mathrm{H} / \alpha$ classification spaces is shown in Figure 3. For unsupervised classification, the classification space is partitioned using a standard set of thresholds that are also shown in Figure 3. The thresholds are somewhat arbitrary and applicationdependent improvements may be possible.

The result of the $\mathrm{H} / \mathrm{A} / \alpha$ classification is then used to initialize a Bayesian minimum-distance classifier based on the complex Wishart distribution of the coherency matrix [5] (this method was initially developed for supervised classification [6]).

It is clear that the full polarimetric information content is used for classification, however, the method is not so much based on a physical interpretation of the data but on similarities of the coherency matrix to a class mean. The result can be further refined using an iterative approach which updates class means after each iteration. This combined, unsupervised algorithm, initially suggested for land applications [5], is used here for sea ice classification for the first time. The final step, the assignment of the derived classes to feature types is a required manual step. Table I lists several polarimetric parameters for each of the 11 classes that were derived for the test scene.

TABLE I

\begin{tabular}{ccccc}
\multicolumn{5}{c}{ Polarimetric Parameters For Class Means } \\
\hline & Anisotropy & Cross Polarized & Ratio & Total Power \\
& $\mathrm{A}$ & $\mathrm{HV}$ & $\mathrm{HH} / \mathrm{VV}$ & \\
Class & 1 & $\mathrm{~dB}$ & $\mathrm{~dB}$ & $\mathrm{~dB}$ \\
\hline Land 1-4 & $\leq 0.33$ & $\geq-20.2$ & $\geq 0.5$ & $\geq-11.1$ \\
Sea ice 1 & 0.54 & -22.9 & -0.2 & -11.1 \\
Sea ice 2 & 0.63 & -23.2 & -0.6 & -12.3 \\
Sea ice 3 & 0.41 & -21.9 & 0.0 & -9.5 \\
Sea ice 4 & 0.66 & -23.1 & -0.3 & -13.4 \\
Water 1 & 0.76 & -24.0 & -1.3 & -14.7 \\
Water 2 & 0.75 & -23.8 & -1.0 & -12.9 \\
Water 3 & 0.70 & -23.9 & -0.9 & -10.7 \\
\hline
\end{tabular}




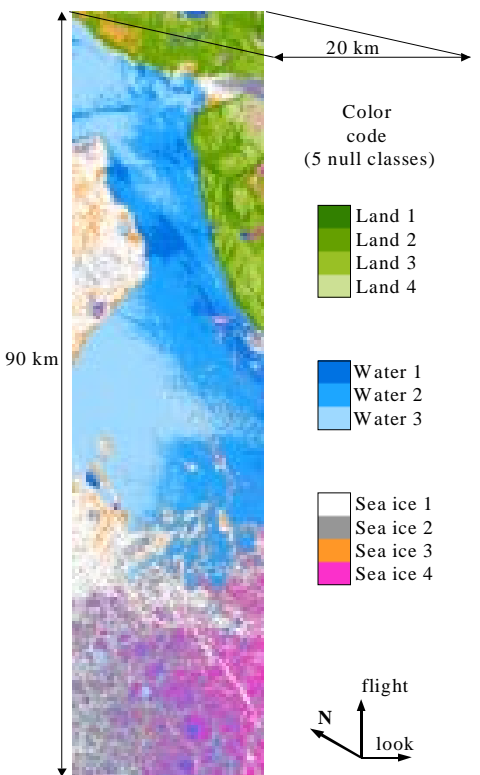

Fig. 4. Classification result after 5 iterations and manual assignment of classes to feature types.

The resulting classification after five iterations is given in Figure 4. The lack of ground truth precludes assigning derived classes to actual ice types; however, analysis of the signatures and spatial distribution of sea ice classes implies deformed sea ice for class Sea ice 3 and thicker sea ice for class Sea ice 1. Classes Sea ice 2 and 4 have properties very similar to water classes thus indicating saturated snow or even surface melt water. Three water classes are derived and strongly correlate to areas with different surface roughness caused by wind.

\section{B. Ice edge detection discriminants}

While RADARSAT-2 will be the first spaceborne sensor capable of providing fully polarimetric data in two of its modes, both RADARSAT-2 and ENVISAT will provide dual polarimetric data. We therefore investigated the potential of single parameters for fast and reliable ice edge detection. The HV channel, available for dual polarimetric modes with one transmit polarization and two receive polarizations, shows separability of sea ice and water at steep incidence angles by applying a threshold of $-23.8 \mathrm{~dB}$. Threshold values were estimated using the rectangular regions shown in Figure 1c. The big advantage of HV is the fact that wind is not an issue for open water surfaces. One reason for this might be the fact that the signal is fairly close to the noise level in this case.

The intensity ratio between the two copolarsized channels, as would be available in alternating polarization mode, is also a candidate for discriminating between the two main feature types (see Figure 1d). The same test fields over open water and sea ice were used to estimate the threshold of $-0.76 \mathrm{~dB}$ between open water and sea ice.

In addition, a threshold of 0.66 was derived for the anisotropy to show the high potential of this parameter for ice
TABLE II

Confusion Matrices For Single Parameter Ice Edge Detection

\begin{tabular}{cccc}
\hline & open water & sea ice & \\
\hline & & & \\
open water & $63.36 \%$ & $7.51 \%$ & $\begin{array}{c}\text { Cross polarized (HV) } \\
\text { sea ice }\end{array}$ \\
(Th $=-23.8 \mathrm{~dB})$
\end{tabular}

edge detection. Table II shows the confusion matrices for water and sea ice as compared to the fully polarimetric result. The anisotropy gives the best performance followed by the HV channel.

\section{CONCLUSIONS}

Multi polarimetric data clearly outperforms single polarization data in its capability for sea ice detection. Automated classification is possible now, and when operational data becomes available, further algorithm improvements will develop. The increased amount of information should allow sea ice type polarimetric signature libraries to be used to initialize the minimum distance classification and thus lead to further automation of the method.

Several single parameters show potential for ice edge detection using simple thresholds. The use of parameter pairs will further improve the results.

\section{ACKNOWLEDGMENTS}

The authors thank Dean Flett from the Canadian Ice Service (CIS) for his valuable comments, and Environment Canada and NASA/JPL for providing data.

\section{REFERENCES}

[1] S. Haykin, E. O. Lews, R. K. Raney and J. R. Rossiter, Remote Sensing of Sea Ice and Icebergs, Wiley, 1994.

[2] L. Eriksson, M. Drinkwater, B. Holt. E. Valjavek and O. Nortier, "SIR-C Polarimetric Radar Results from the Weddell Sea, Antarctica," Proc. IGARSS'98, 1998.

[3] F. T. Ulaby and C. Elachi, Radar Polarimetry for Geoscience Applications, Artech House, 1990.

[4] S. R. Cloude and E. Pottier, "An Entropy Based Classification Scheme for Land Applications of Polarimetric SAR," IEEE Trans. Geosci. Remote Sensing, vol. 35, no. 1, pp. 68-78, January 1997

[5] E. Pottier and J.-S. Lee, "Application of the H/A/ $\alpha$ Polarimetric Decomposition Theorem for Unsupervised Classification of Fully Polarimetric SAR Data Based on the Wishart Distribution," in Proc. CEOS'99, 1999.

[6] J.-S. Lee, M. Grunes and R. Kwok, "Classification of multilook polarimetric SAR imagery based on complex Wishart distribution," Int. J. Remote Sensing, vol. 15, no. 11, pp. 22992311, 1994. 